\title{
Effective Preparation of Cycloheptimidazol-4-one Compounds
}

\author{
Motoharu Sonegawa, * Yoshinori Iwai, Hiroshi Tomiyama, and Tsuyoshi TomiYama \\ Kotobuki Research Laboratories, Kotobuki Seiyaku Company, Ltd.; 6351 Sakaki-Machi, Nagano 389-0697, Japan. \\ Received October 29, 2005; accepted February 2, 2006; published online February 7, 2006
}

Effective preparation of cycloheptimidazol-4-ones was developed. The reactions of 2-tosyloxytropone (5) with amidines (6) carried out under simple conditions such as aq. $\mathrm{NaOH}$ in toluene at $35^{\circ} \mathrm{C}$ afforded the corresponding cycloheptimidazol-4-ones (3) in low yield. However, by adding tetra- $n$-butylammonium bromide ( $n$ $\mathrm{Bu}_{4} \mathrm{NBr}$ ) to this reaction system, the yield was improved dramatically. The reaction conditions were screened in detail.

Key words cycloheptimidazolone; phase transfer catalyst (PTC); angiotensin II

The renin-angiotensin system (RAS) plays a critical role in blood pressure control. ${ }^{1)}$ Angiotensin II (AII), the active hormone generated in RAS, shows a potent vasoconstrictive effect which is thought to be the etiology of hypertension and congestive heart failure. Blocking the action of AII at the AII receptor level is a more effective approach and considerable effort has been made in the search for nonpeptide AII receptor antagonists in recent years.

We have recently described the identification of Pratosartan and KT3-866, which are orally active, competitive AII receptor antagonists. ${ }^{2,3)}$ Tellew et al. have reported that compound (1) acts as a dual antagonist both of AII and endothelin subtype $\mathrm{A}\left(\mathrm{ET}_{\mathrm{A}}\right){ }^{4}{ }^{4}$ These antagonists include a 5,6,7,8tetrahydrocycloheptimidazole skeleton (2) as a major component (Chart 1). Therefore, 5,6,7,8-tetrahydrocycloheptimidazole is considered to be a biologically important molecular part. 5,6,7,8-Tetrahydrocycloheptimidazol-4-one (4), in the case of $\mathrm{X}=\mathrm{O}$ in the skeleton (2), could be easily obtained from cycloheptimidazol-4-one (3) by partial hydrogenation (Chart 2).

Cycloheptimidazol-4-one compound (3a) has been hitherto prepared by the treatment of 2-tosyloxytropone (5a) with acetamidine under alkaline reaction condition (Chart

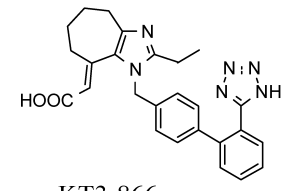

KT3-866

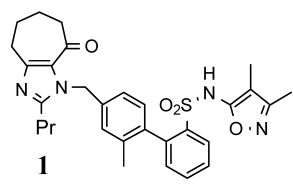

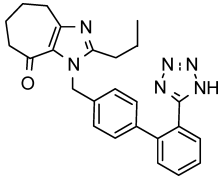

Pratosartan (KT3-671)

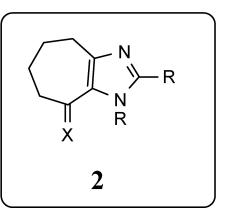

Chart 1. Structure of Biological Active Tetrahydrocycloheptimidazole Analogs

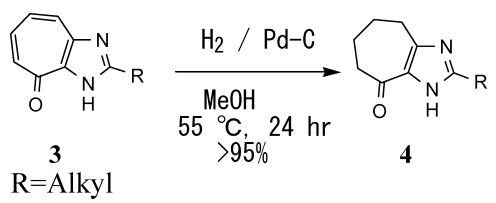

Chart 2. Preparation of Tetrahydrocycloheptimidazol-4-one (4)
3). ${ }^{5)}$ It seems that in this homogeneous reaction system hydrolysis of the substrates occurs. Therefore, the yield was not always satisfactory. Another problem in the industrial-scale manufacture would be not only how to achieve easy recovery of the solvents, but also to avoid highly inflammable operation. In order to prevent the hydrolysis and to use recoverable solvents, biphasic reaction conditions were examined. The cyclization of 2-tosyloxytropone with amidine in the combination of aq. $\mathrm{NaOH}$ and toluene provided cycloheptimidazol4 -one in only $16 \%$ yield (Table 1 ). The details of the reaction conditions were examined because better yield was obtained when phase transfer catalyst (tetra- $n$-butylammonium bromide $\left.\left(n-\mathrm{Bu}_{4} \mathrm{NBr}\right)\right)$ was added to this reaction mixture.

\section{Results and Discussion}

In the first step of examination of reaction conditions, influence of the aqueous alkaline solution in order to decrease the formation of different products was checked (Table 2). ${ }^{6,7)}$ By changing the base concentration with the constant equivalent of the base against $5 \mathbf{a}$, the best yield was observed in the use of $30 \%$ aqueous $\mathrm{NaOH}$ solution. $\mathrm{NaOH}$ showed the most efficacy among alkali hydroxides under these conditions.

In the second step, reaction temperature was tested because it could be an important factor to control the produc-

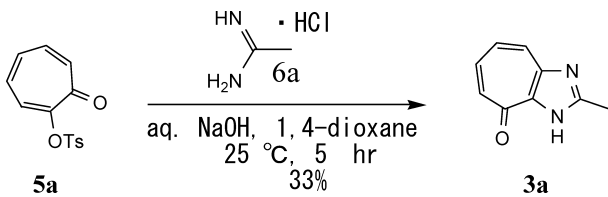

Chart 3. Preparation of 2-Methyl-3H-cycloheptimidazol-4-one (3a) in aq. $\mathrm{NaOH} / 1,4-$ Dioxane

Table 1. Reaction of $n$-Butyramidine (6c) with Tosyloxytropone (5a)

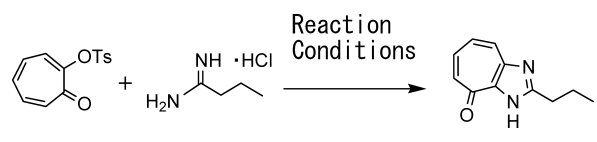

$5 a$

\begin{tabular}{clc}
\hline \hline Entry & \multicolumn{1}{c}{ Reaction conditions } & $\begin{array}{c}\text { Yield }^{a} \\
(\%)\end{array}$ \\
\hline 1 & $20 \%$ aq. $\mathrm{NaOH}$, toluene, $30^{\circ} \mathrm{C}, 9 \mathrm{~h}$ & 16 \\
2 & $20 \%$ aq. $\mathrm{NaOH}, n-\mathrm{Bu}_{4} \mathrm{NBr}(0.4 \mathrm{eq})$, toluene, $20^{\circ} \mathrm{C}, 18 \mathrm{~h}$ & 53
\end{tabular}

a) Isolated yield of the compound (3c). ${ }^{2)}$ 
Table 2. Effect of Concentration of Aqueous Alkaline Solution

\begin{tabular}{|c|c|c|c|}
\hline \multicolumn{4}{|c|}{ Base } \\
\hline $5 \mathbf{a}$ & \multicolumn{3}{|c|}{$n-\mathrm{Bu}_{4} \mathrm{NBr}$ (0.4eq.), toluene, $20{ }^{\circ} \mathrm{C}$} \\
\hline Entry & Base & $\begin{array}{l}\text { Reaction time } \\
\text { (h) }\end{array}$ & $\begin{array}{l}\left.\text { Yield }^{a}\right) \\
(\%)\end{array}$ \\
\hline 1 & $10 \%$ aq. $\mathrm{NaOH}$ & 24 & 25 \\
\hline 2 & $20 \%$ aq. $\mathrm{NaOH}$ & 18 & 53 \\
\hline 3 & $30 \%$ aq. $\mathrm{NaOH}$ & 10 & 72 \\
\hline 4 & $40 \%$ aq. $\mathrm{NaOH}$ & 8 & 68 \\
\hline 5 & $50 \%$ aq. $\mathrm{NaOH}$ & 5 & 62 \\
\hline 6 & $30 \%$ aq. $\mathrm{KOH}$ & 10 & 64 \\
\hline 7 & $30 \%$ aq. $\mathrm{LiOH}$ & 12 & 65 \\
\hline
\end{tabular}

a) Isolated yield of the compound $(\mathbf{3 c})^{2)}$.

Table 3. Effect of Reaction Temperature

\begin{tabular}{|c|c|c|c|c|}
\hline Entry & $\mathbf{6 c}$ equiv. $v s . \mathbf{5 a}$ & $\begin{array}{l}\text { Temp. } \\
\left({ }^{\circ} \mathrm{C}\right)\end{array}$ & $\begin{array}{l}\text { Reaction time } \\
\text { (h) }\end{array}$ & $\begin{array}{c}\text { Yield }^{a)} \\
(\%)\end{array}$ \\
\hline 1 & 1.0 & 20 & 8 & 57 \\
\hline 2 & 1.0 & 30 & 8 & 62 \\
\hline 3 & 1.0 & 40 & 8 & 61 \\
\hline 4 & 1.0 & 50 & 8 & 50 \\
\hline 5 & 1.5 & 20 & 5 & 72 \\
\hline 6 & 1.5 & 30 & 5 & 82 \\
\hline 7 & 1.5 & 40 & 5 & 76 \\
\hline 8 & 1.5 & 50 & 5 & 65 \\
\hline
\end{tabular}

a) Isolated yield of the compound $(\mathbf{3 c})^{2)}$.

tion of $\mathbf{3 c}$ and decomposition of substrates (Table 3 ). The highest yield was observed at $30^{\circ} \mathrm{C}$ in the range of 20 $50^{\circ} \mathrm{C}$. In addition, the use of $1.5 \mathrm{eq}$ of the amidine $(6 \mathrm{c}) \mathrm{re}-$ sulted in better yield.

Next, the effectiveness of several kinds of phase transfer catalyst was examined (Table 4). A similar yield was obtained in the phase transfer catalyst that consists of a butyl group. However, when a tetra- $n$-hexylammonium bromide $\left(n-\mathrm{Hex}_{4} \mathrm{NBr}\right)$ was used, the accurate isolation yield was not obtained because the $n-\mathrm{Hex}_{4} \mathrm{NBr}$ was contaminated into the products. When 0.2 eq of the phase transfer catalyst was used in this reaction, prolonged reaction time was required.

Various kinds of amidines were treated with 2-tosyloxytropone (5a) and butyramidine was reacted with 2-tosyloxytropones $(\mathbf{5 b}$ or $\mathbf{5 c})$ under optimized reaction conditions (Table $5)$. The reactions with acetamidine or propionamidine afforded the corresponding products in modest yields. In contrast, use of benzamidine and pentanamidine resulted in even higher yields. This would be considered due to the lipophilicity of the amidines, because the lipophilic amidine increases the solubility to toluene.

The reaction mechanism of this interesting cyclization is demonstrated in Chart 4. It should be noted that if the same reaction with 2-chlorotropone instead of 2-tosyloxytropone is carried out under similar conditions, no expected products were obtained. On the other hand, the reaction with 4-isopropyl-2-tosyloxytropone (5b) afforded not only 6-isopropylcycloheptimidazol-4-one (3h), but also $p$-toluenesulfinic acid
Table 4. Effect of Phase Transfer Catalyst

$$
\mathbf{5 a}+\mathbf{6 c} \stackrel{30 \% \text { aq. } \mathrm{NaOH}}{\stackrel{\text { PTC (0.4eq.) }}{\text { toluene, } 30{ }^{\circ} \mathrm{C}}} \mathbf{3 c}
$$

\begin{tabular}{clcc}
\hline \hline Entry & $\begin{array}{c}\text { Phase transfer } \\
\text { catalyst (PTC) }\end{array}$ & $\begin{array}{r}\text { Reaction time } \\
(\mathrm{h})\end{array}$ & $\begin{array}{c}\text { Yield }^{a)} \\
(\%)\end{array}$ \\
\hline 1 & $n-\mathrm{Bu}_{4} \mathrm{NCl}$ & 5 & 80 \\
2 & $n-\mathrm{Bu}_{4} \mathrm{NBr}$ & 5 & 82 \\
3 & $n-\mathrm{Bu}_{4} \mathrm{NI}$ & 5 & 74 \\
4 & $n-\mathrm{Et}_{4} \mathrm{NBr}$ & 40 & 52 \\
5 & $n-\mathrm{Pr}_{4} \mathrm{NBr}$ & 8 & 69 \\
6 & $n-\mathrm{Hex}_{4} \mathrm{NBr}$ & 1 & $-b)$
\end{tabular}

a) Isolated yield of the compound $(3 \mathbf{c})^{2)}$ b) Mixture with $n$ - $\mathrm{Hex}_{4} \mathrm{NBr}$ was obtained.

Table 5. Reaction of Amidines (6) with 2-Tosyloxytropones (5)

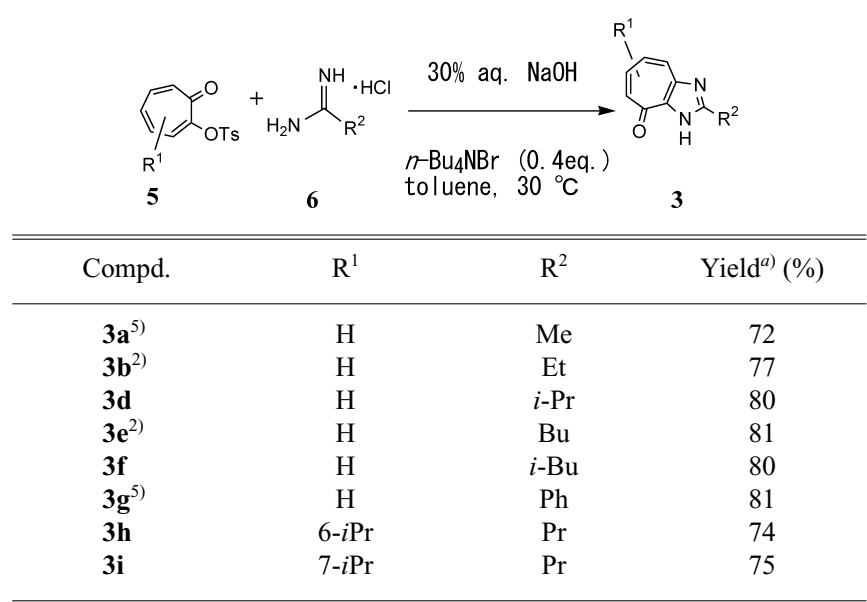

a) Isolated yield of the compound (3)

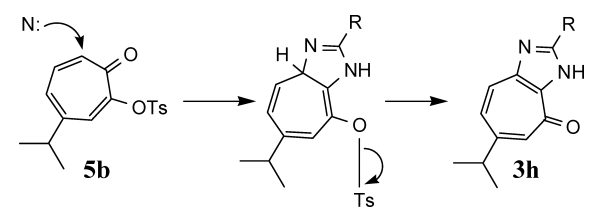

Chart 4. Reaction Mechanism for the Formation of Cycloheptimidazol-4one

liberated from the reaction ${ }^{8)}$ and besides, a small amount of 5-isopropylcycloheptimidazole as a by-product was obtained. From these results, it could be considered that the cyclization occurs after an anion of amidine attacks at the 7-position of tosyloxytropone and then followed by the cleavage of the $\mathrm{O}-\mathrm{S}$ bond takes place preferentially similar to the formation of 2-amino-3-carbamoylcyclohepta[b]furan-8-one. ${ }^{9)}$

Regioselective alkylation to cycloheptimidazol-4-ones reported in this paper provided Pratosartan as shown in the successive paper. ${ }^{10)}$

\section{Conclusion}

The reaction of 2-tosyloxytropone with amidines in the presence of $n-\mathrm{Bu}_{4} \mathrm{NBr}$ as a phase transfer catalyst in aq. $\mathrm{NaOH} /$ toluene under industrially useful conditions provided cycloheptimidazol-4-ones in high yield. 


\section{Experimental}

Melting points were determined on Yamato melting point apparatus and are uncorrected. Infrared (IR) spectra were recorded on a Hitachi 270-30. Proton nuclear magnetic resonance ( $\left.{ }^{1} \mathrm{H}-\mathrm{NMR}\right)$ spectra were measured at $400 \mathrm{MHz}$ on a JEOL EX-400 Fourier-transform NMR spectrometer. Chemical shifts are quoted in part per million (ppm) with tetramethylsilane as an internal standard. Coupling constants $(J)$ are given in $\mathrm{Hz}$. The following abbreviations are used: $\mathrm{s}$, singlet; $\mathrm{d}$, doublet; $\mathrm{t}$, triplet; $\mathrm{q}$, quartet; br s, broad singlet; dd, doublet of doublet; $\mathrm{m}$, multiplet. Mass spectra (MS) were taken on a Hitachi M-80B spectrometer. For column chromatography, silica gel (Merck, Kieselgel 60, 70-230 mesh) was used.

2-Propyl-5,6,7,8-tetrahydro-3 $\boldsymbol{H}$-cycloheptimidazol-4-one $(\mathbf{4 c})^{2)}$ To a solution of 2-propyl-3H-cycloheptimidazol-4-one $(3 \mathbf{c}, 4.00 \mathrm{~g})$ in $\mathrm{MeOH}$ $(80 \mathrm{ml})$ was added $10 \% \mathrm{Pd}$ on carbon $(0.40 \mathrm{~g})$ and the mixture was stirred for $24 \mathrm{~h}$ under an atmosphere of hydrogen $(1 \mathrm{~atm})$ at $55^{\circ} \mathrm{C}$. After filtration of the catalyst, the filtrate was concentrated under the reduced pressure. Then, the residue was purified by silica gel column chromatography (EtOAc $\mathrm{MeOH}=20 / 1)$, affording $4 \mathbf{c}(4.03 \mathrm{~g}, 99 \%)$ as a colorless solid. Recrystallization from EtOAc gave colorless crystals. mp $76-77^{\circ} \mathrm{C}$. IR $(\mathrm{KBr}) \mathrm{cm}^{-1}$ : $3236,2916,1608,1542,1522,1458,1408 .{ }^{1} \mathrm{H}-\mathrm{NMR}\left(\mathrm{CDCl}_{3}\right) \delta: 0.98(3 \mathrm{H}, \mathrm{t}$, $J=7.2 \mathrm{~Hz}), 1.77(2 \mathrm{H}, \mathrm{m}), 1.88-2.08(4 \mathrm{H}, \mathrm{m}), 2.65-2.75(4 \mathrm{H}, \mathrm{m}), 2.99$ $(2 \mathrm{H}, \mathrm{t}, J=7.2 \mathrm{~Hz}), 10.10-10.40(1 \mathrm{H}, \mathrm{br}) . \mathrm{MS} m / z: 192\left(\mathrm{M}^{+}\right), 177,164$ (B.P.).

General Procedure for 2-Propyl-6-isopropyl-3H-cycloheptimidazol-4one (3h) To a mixture of $30 \% \mathrm{NaOH}(6.85 \mathrm{~g})$ and toluene $(25 \mathrm{ml}), 4$-isopropyl-2-tosyloxytropone $(\mathbf{5 b}, 3.18 \mathrm{~g})$, butyramidine hydrochloride $(\mathbf{6 c}$ $1.84 \mathrm{~g})$ and tetra- $n$-butylammonium bromide $(1.61 \mathrm{~g})$ were added successively and the mixture was stirred at $30^{\circ} \mathrm{C}$ for $5 \mathrm{~h}$. The reaction mixture was treated with excess saturated aqueous ammonium chloride and extracted with EtOAc $(3 \times 20 \mathrm{ml})$. The combined extract layers were washed with brine and dried over $\mathrm{Na}_{2} \mathrm{SO}_{4}$. The solvent was removed under the reduced pressure. Then, the residue was purified by silica gel column chromatography $(\mathrm{EtOAc} / \mathrm{MeOH}=50 / 1)$, affording $3 \mathrm{~h}(1.71 \mathrm{~g}, 74 \%)$. Recrystallization from $i \mathrm{PrOH}$ gave pale yellow needles. mp $166-168^{\circ} \mathrm{C}$. IR $(\mathrm{KBr}) \mathrm{cm}^{-1}: 3064$, $2944,1620,1575,1527,1491 .{ }^{1} \mathrm{H}-\mathrm{NMR}\left(\mathrm{CDCl}_{3}\right) \delta: 1.03(3 \mathrm{H}, \mathrm{t}, J=7.2 \mathrm{~Hz})$ $1.31(6 \mathrm{H}, \mathrm{d}, J=6.8 \mathrm{~Hz}), 1.91(2 \mathrm{H}, \mathrm{m}), 2.85-2.98(1 \mathrm{H}, \mathrm{m}), 2.99(2 \mathrm{H}, \mathrm{d}$, $J=7.4 \mathrm{~Hz}), 7.02(1 \mathrm{H}, \mathrm{d}, J=11.6 \mathrm{~Hz}), 7.25(1 \mathrm{H}, \mathrm{s}), 7.77(1 \mathrm{H}, \mathrm{d}, J=11.6 \mathrm{~Hz})$ MS $m / z: 230\left(\mathrm{M}^{+}\right), 202$ (B.P.), 187

2-Methyl-3H-cycloheptimidazol-4-one (3a): $\mathrm{mp}$ 191-193 ${ }^{\circ} \mathrm{C}(i \mathrm{PrOH}$, pale yellow needles, Lit. $\left.191^{\circ} \mathrm{C}\right)$. IR $(\mathrm{KBr}) \mathrm{cm}^{-1}: 3076,1623,1572,1530$, $1488,1293,1272 .{ }^{1} \mathrm{H}-\mathrm{NMR}\left(\mathrm{CDCl}_{3}\right) \delta: 2.75(3 \mathrm{H}, \mathrm{s}), 7.10(1 \mathrm{H}, \mathrm{dd}, J=8.8$, $11.2 \mathrm{~Hz}), 7.35(1 \mathrm{H}, \mathrm{d}, J=12.0 \mathrm{~Hz}), 7.43-7.49(1 \mathrm{H}, \mathrm{m}), 7.85(1 \mathrm{H}, \mathrm{d}$, $J=11.2 \mathrm{~Hz}$ ). MS $m / z: 160\left(\mathrm{M}^{+}\right), 132$ (B.P.), 63.

2-Ethyl-3H-cycloheptimidazol-4-one (3b): mp $160-164^{\circ} \mathrm{C}(i \mathrm{PrOH}$, pale yellow needles). IR (KBr) cm ${ }^{-1}: 3070,1620,1566,1527,1488,1281 .{ }^{1} \mathrm{H}-$ NMR $\left(\mathrm{CDCl}_{3}\right) \delta: 1.48(3 \mathrm{H}, \mathrm{t}, J=7.6 \mathrm{~Hz}), 3.07(2 \mathrm{H}, \mathrm{t}, J=7.6 \mathrm{~Hz}), 7.08(1 \mathrm{H}$, dd, $J=8.8,10.8 \mathrm{~Hz}), 7.33(1 \mathrm{H}, \mathrm{d}, J=12.0 \mathrm{~Hz}), 7.41-7.49(1 \mathrm{H}, \mathrm{m}), 7.86$ $(1 \mathrm{H}, \mathrm{d}, J=11.2 \mathrm{~Hz})$. MS $m / z: 174\left(\mathrm{M}^{+}\right.$, B.P.), 145,63

2-Propyl-3 $H$-cycloheptimidazol-4-one $(3 \mathrm{c}): \mathrm{mp} \quad 142-144^{\circ} \mathrm{C} \quad(i \mathrm{PrOH}$, pale yellow needles). IR (KBr) cm $\mathrm{cm}^{-1}: 3070,1617,1569,1524,1485,1269$. ${ }^{1} \mathrm{H}-\mathrm{NMR}\left(\mathrm{CDCl}_{3}\right) \delta: 1.04(3 \mathrm{H}, \mathrm{t}, J=7.2 \mathrm{~Hz}), 1.82-1.99(2 \mathrm{H}, \mathrm{m}), 3.01(2 \mathrm{H}$, t, $J=7.6 \mathrm{~Hz}), 7.08(1 \mathrm{H}, \mathrm{dd}, J=8.8,11.2 \mathrm{~Hz}), 7.31(1 \mathrm{H}, \mathrm{d}, J=12.4 \mathrm{~Hz})$, $7.40-7.49(1 \mathrm{H}, \mathrm{m}), 7.85(1 \mathrm{H}, \mathrm{d}, J=11.2 \mathrm{~Hz})$. MS $m / z: 188\left(\mathrm{M}^{+}\right), 173,160$ (B.P.).

2-Isopropyl-3H-cycloheptimidazol-4-one (3d): $\mathrm{mp} 183-186^{\circ} \mathrm{C}(i \mathrm{PrOH}$, pale yellow needles). IR (KBr) cm ${ }^{-1}: 3070,1620,1572,1521,1491,1275$
${ }^{1} \mathrm{H}-\mathrm{NMR}\left(\mathrm{CDCl}_{3}\right) \delta: 1.50(6 \mathrm{H}, \mathrm{d}, J=7.2 \mathrm{~Hz}), 3.39(1 \mathrm{H}, \mathrm{m}), 7.07(1 \mathrm{H}, \mathrm{dd}$, $J=8.8,11.2 \mathrm{~Hz}), 7.30(1 \mathrm{H}, \mathrm{d}, J=12.0 \mathrm{~Hz}), 7.40-7.46(1 \mathrm{H}, \mathrm{m}), 7.86(1 \mathrm{H}, \mathrm{d}$ $J=10.8 \mathrm{~Hz})$. MS $m / z: 188\left(\mathrm{M}^{+}\right), 173$ (B.P.), 145.

2-Butyl-3H-cycloheptimidazol-4-one (3e): $\mathrm{mp} 132-134{ }^{\circ} \mathrm{C}(i \mathrm{PrOH}$, pale yellow needles). IR (KBr) cm ${ }^{-1}: 3070,2938,1620,1575,1524,1488 .{ }^{1} \mathrm{H}-$ NMR $\left(\mathrm{CDCl}_{3}\right) \delta: 0.96(3 \mathrm{H}, \mathrm{t}, J=7.2 \mathrm{~Hz}), 1.40-1.51(2 \mathrm{H}, \mathrm{m}), 1.82-1.91$ $(2 \mathrm{H}, \mathrm{m}), 3.03(2 \mathrm{H}, \mathrm{t}, J=7.6 \mathrm{~Hz}), 7.07(1 \mathrm{H}, \mathrm{dd}, J=8.8,10.8 \mathrm{~Hz}), 7.30(1 \mathrm{H}, \mathrm{d}$, $J=12.4 \mathrm{~Hz}), 7.40-7.47(1 \mathrm{H}, \mathrm{m}), 7.85(1 \mathrm{H}, \mathrm{d}, J=11.2 \mathrm{~Hz}) . \mathrm{MS} m / z: 202$ $\left(\mathrm{M}^{+}\right), 173,160$ (B.P.)

2-Isobutyl-3H-cycloheptimidazol-4-one (3f): $\mathrm{mp} 153-154{ }^{\circ} \mathrm{C}(i \mathrm{PrOH}$, pale yellow needles). IR (KBr) cm ${ }^{-1}: 3070,2944,1620,1569,1524,1488$, 1272. ${ }^{1} \mathrm{H}-\mathrm{NMR}\left(\mathrm{CDCl}_{3}\right) \delta: 1.02(6 \mathrm{H}, \mathrm{d}, J=6.4 \mathrm{~Hz}), 2.29(1 \mathrm{H}, \mathrm{m}), 2.89(2 \mathrm{H}$, d, $J=7.4 \mathrm{~Hz}), 7.08(1 \mathrm{H}, \mathrm{dd}, J=8.8,11.2 \mathrm{~Hz}), 7.30(1 \mathrm{H}, \mathrm{d}, J=12.4 \mathrm{~Hz}), 7.44$ $(1 \mathrm{H}, \mathrm{dd}, J=8.8,12.4 \mathrm{~Hz}), 7.85(1 \mathrm{H}, \mathrm{d}, J=11.2 \mathrm{~Hz})$. MS $m / z: 202\left(\mathrm{M}^{+}\right), 187$, 160 (B.P.).

2-Phenyl-3H-cycloheptimidazol-4-one (3g): $\mathrm{mp} 227-230^{\circ} \mathrm{C}(i \mathrm{PrOH}$, pale yellow needles, Lit. $\left.212^{\circ} \mathrm{C}\right)$. IR $(\mathrm{KBr}) \mathrm{cm}^{-1}: 3040,1623,1569,1545$, $1464,1278 .{ }^{1} \mathrm{H}-\mathrm{NMR}\left(\mathrm{CDCl}_{3}\right) \delta: 7.12(1 \mathrm{H}, \mathrm{dd}, J=8.4,10.8 \mathrm{~Hz}), 7.36(1 \mathrm{H}$, d, $J=12.0 \mathrm{~Hz}), 7.47(1 \mathrm{H}, \mathrm{dd}, J=8.4,12.0 \mathrm{~Hz}), 7.51-7.56(3 \mathrm{H}, \mathrm{m}), 7.95$ $(1 \mathrm{H}, \mathrm{d}, J=10.8 \mathrm{~Hz}), 8.39(2 \mathrm{H}, \mathrm{d}, J=8.0 \mathrm{~Hz})$. MS $m / z: 222\left(\mathrm{M}^{+}\right), 194$ (B.P.), 64.

2-Propyl-7-isopropyl-3H-cycloheptimidazol-4-one (3i): $\mathrm{mp} 135-136^{\circ} \mathrm{C}$ ( $i \mathrm{PrOH}$, pale yellow needles). IR $(\mathrm{KBr}) \mathrm{cm}^{-1}: 3064,2944,1623,1572$, $1515,1482 .{ }^{1} \mathrm{H}-\mathrm{NMR}\left(\mathrm{CDCl}_{3}\right) \delta: 1.03(3 \mathrm{H}, \mathrm{t}, J=7.2 \mathrm{~Hz}), 1.31(6 \mathrm{H}, \mathrm{d}$, $J=6.8 \mathrm{~Hz}), \quad 1.82-1.99(2 \mathrm{H}, \mathrm{m}), 2.80-2.99(1 \mathrm{H}, \mathrm{m}), 2.98(2 \mathrm{H}, \mathrm{t}$, $J=7.6 \mathrm{~Hz}), 7.28(1 \mathrm{H}, \mathrm{d}, J=12.8 \mathrm{~Hz}), 7.38(1 \mathrm{H}, \mathrm{d}, J=12.8 \mathrm{~Hz}), 7.75(1 \mathrm{H}, \mathrm{s})$. MS $m / z: 230\left(\mathrm{M}^{+}\right), 215,202$ (B.P.).

Acknowledgments We thank Professor S. Ikegami, Teikyo University, for his generous and helpful discussions.

\section{References and Notes}

1) Duncia J. V., Chiu A. T., Carini D. J., Gregory G. B., Johnson A. L., Price W. A., Wells G. J., Wong P. C., Calabrese J. C., Timmermans P. B. M. W. M., J. Med. Chem., 33, 1312-1329 (1990).

2) Yanagisawa T., Ueyama N., Kawai T., Sonegawa M., Baba H., Mochizuki S., Kosakai K., Tomiyama T., Bioorg. Med. Chem. Lett., 3, 1559-1564 (1993).

3) Ueyama N., Yanagisawa T., Baba H., Kuroiwa K., Hayashi H., Sonegawa M., Tomiyama T., Bioorg. Med. Chem. Lett., 4, 1637-1642 (1994).

4) Tellew J. E., Baska R. A. F., Beyer S. M., Carlson K. E., Cornelius L. A., Fadnis L., Gu Z., Kunst B. L., Kowala M. C., Monshizadegan H., Murugesan N., Ryan C. S., Valentine M. T., Yang Y., Macor J. E., Bioorg. Med. Chem. Lett., 13, 1093-1096 (2003).

5) Nakazawa J., Sato Y., Soma N., Sankyou Kenkyusyo Nenpo, 21, 4756 (1969)

6) Nozoe T., Kagakunoryouiki, 17, 831-849 (1963).

7) Yanagisawa T., Kosakai K., Tomiyama T., Yasunami M., Takase K., Chem. Pharm. Bull., 38, 3355-3358 (1990).

8) A precipitate which was obtained from the acidified reaction mixture showed $\mathrm{m} / z 156$ mass of $p$-toluensulfinic acid.

9) Nozoe T., Takase K., Kato M., Nogi T., Tetrahedron, 27, 6023-6035 (1971).

10) Sonegawa M., Yokota M., Tomiyama H., Tomiyama T., Chem. Pharm. Bull., 54, 706-710 (2006). 